\title{
Association of the matrix metalloproteinase-3 polymorphisms rs679620 and rs3025058 with ischemic stroke risk: a meta-analysis
}

This article was published in the following Dove Press journal:

Neuropsychiatric Disease and Treatment

\section{Qi-Wei Zhang}

Department of Neurosurgery, The Affiliated Hospital of Jilin Medical University, Jilin, People's Republic of China
Correspondence: Qi-Wei Zhang

Department of Neurosurgery,

The Affiliated Hospital of Jilin Medical University, No 8I Huashan Road, Jilin, People's Republic of China

Tel +8643264560740

Email 1980wei0242@sina.com
Purpose: The relationship of the matrix metalloproteinase-3 (MMP-3) polymorphisms rs679620 and rs3025058 with ischemic stroke has received much attention. The aim of the present study was to perform a meta-analysis of published case-control studies to evaluate the cumulative evidence.

Methods: We performed a search of ISI Web of Science, Embase, PubMed, and China National Knowledge Infrastructure databases. Pooled odds ratios (ORs) were appropriately derived from fixed-effects or random-effects models.

Results: We identified seven eligible studies including 5,204 subjects. The pooled analysis showed that the MMP-3 rs679620 A allele carriers had increased risk of ischemic stroke compared with homozygotes for the G allele in Asians (AA + GA vs GG: OR $=1.42,95 \%$ CI: $1.05-1.91, P=0.022$ ). Concerning the rs 3025058 polymorphism, the results did not suggest an association between rs3025058 genotypes and ischemic stroke risk $(5 \mathrm{~A} 5 \mathrm{~A}+6 \mathrm{~A} 5 \mathrm{~A}$ vs $6 \mathrm{~A} 6 \mathrm{~A}$ : $\mathrm{OR}=1.04,95 \% \mathrm{CI}: 0.73-1.47, P=0.844 ; 5 \mathrm{~A} 5 \mathrm{~A}$ vs $6 \mathrm{~A} 5 \mathrm{~A}+6 \mathrm{~A} 6 \mathrm{~A}: \mathrm{OR}=1.14,95 \%$ CI: $0.74-1.77$, $P=0.556$; and 5A5A vs 6A6A: $\mathrm{OR}=1.11,95 \% \mathrm{CI}: 0.68-1.80, P=0.677)$. In subgroup analysis by ethnicity, no statistically significant associations were demonstrated for rs3025058 in Asians and Caucasians, respectively. There was no evidence for publication bias.

Conclusion: Our findings indicate that the rs679620 A allele carriers have increased risk of ischemic stroke in Asians, but there is no association between rs3025058 and ischemic stroke risk.

Keywords: ischemic stroke, meta-analysis, MMP-3, polymorphism

\section{Introduction}

Stroke is the third largest cause of death and a leading cause of short-term and long-term disabilities in the developing world. ${ }^{1}$ Annually, an estimated 795,000 new and recurrent strokes affect people in the USA. ${ }^{2}$ The financial burden of stroke is huge; acute and long-term treatments for stroke patients consume $>5 \%$ of many countries' health care budget. ${ }^{3}$ The majority ( $\left.75 \%-80 \%\right)$ of strokes are ischemic in nature, resulting from an obstruction within a blood vessel supplying blood to parts of the brain. ${ }^{4}$ Conventional risk factors including hypertension, obesity, type 2 diabetes, cigarette smoking, and atrial fibrillation fail to explain all stroke risk. Accumulating evidence from genetic studies suggests that genetic factors may account for some of this unexplained risk. ${ }^{5}$

Matrix metalloproteinases (MMPs) are a family of calcium- and zinc-dependent proteolytic enzymes that cleave molecules from the extracellular matrix and basement membranes. ${ }^{6}$ MMP-3 (stromelysin-1) is a key member of the MMP family. It can degrade the major components of the extracellular matrix and is also capable of 
degrading fibronectin, laminin, and cartilage proteoglycans. ${ }^{7}$ In situ hybridization analysis showed that MMP-3 was overexpressed in smooth muscle cells and large clusters of macrophages that contained intracellular lipid deposits within atherosclerotic plaques. ${ }^{8}$ In addition, increased expression of MMP-3 was observed in human atherosclerotic plaques' shoulders and regions of foam cell accumulation. ${ }^{9}$ Blood levels of active MMP-3 were associated with the extent of carotid atherosclerosis. ${ }^{10}$ It is now widely accepted that deregulation of MMP-3 plays a pivotal role in vascular remodeling and carotid atherosclerosis development.

In humans, the $M M P-3$ gene is located on chromosome 11. Among the variants identified in $M M P$-3, two polymorphisms have been frequently studied. The rs679620 polymorphism is a guanine $(\mathrm{G})$ to adenine $(\mathrm{A})$ transition at nucleotide position 28 within exon $2 .{ }^{11}$ The rs3025058 polymorphism in the promoter region is an insertion/deletion variant characterized by the presence of either five or six adenine residues, with the $5 \mathrm{~A}$ allele having greater promoter activity than the 6 A allele. ${ }^{12}$ Previous association studies have evaluated the association of rs679620 and rs3025058 with ischemic stroke risk. However, these studies may have been underpowered to detect the true relationship or varied markedly in the populations studied and the sampling strategies. Our aim was to validate the reported association of these two polymorphisms with risk of ischemic stroke in a meta-analysis of published case-control studies.

\section{Methods}

\section{Search strategy}

We followed the Preferred Reporting Items for Systematic Reviews and Meta-Analysis (PRISMA). Because we synthesized data from previously published publications, the metaanalysis was exempt from ethics approval. We searched ISI Web of Science (http://www.webofknowledge.com), Embase (http://www.embase.com), PubMed (http://www.pubmed.

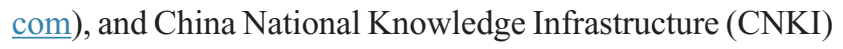
(http://www.cnki.net) databases to identify case-control studies containing data on the relationship of rs679620 and rs3025058 with risk of ischemic stroke using the following keywords: stroke, ischemic stroke, cerebral infarction, polymorphism, matrix metalloproteinase-3, rs3025058, rs679620, gene, and risk. Search dates ranged from January 1995 to September 2017. Additional studies were searched through screening reference lists of retrieved studies, but no additional articles were identified using this strategy. A search of the gray area literature was not performed.

\section{Inclusion/exclusion criteria and data extraction}

Any study was considered to be eligible for inclusion in the meta-analysis if it met the following criteria: 1) case-control studies reporting data for the association of rs3025058 and/or rs679620 with ischemic stroke risk and 2) studies published in English or Chinese. Experimental studies, reviews, and meeting abstracts were excluded. The following information was abstracted from the study reports: journal name, first author's last name, year of publication, country, ethnicity of the study populations, the number of subjects in both cases and controls, characteristics of ischemic stroke patients, source of controls, and method by which the polymorphism was investigated. Study quality was assessed using the Newcastle-Ottawa Scale (NOS) quality assessment scale for case-control studies. ${ }^{13}$ No studies were excluded according to NOS scores.

\section{Statistical analyses}

Statistical software STATA version 11.0 (StataCorp LP, College Station, TX, USA) was used to conduct metaanalyses. A $P$-value of $<0.05$ was assumed to be statistically significant, except for heterogeneity where $P<0.10$ was used. The combined effect (expressed as the odds ratio [OR] with 95\% CI) for the association of rs679620 and rs3025058 with risk of ischemic stroke was calculated using the random-effects (DerSimonian Laird) model or the fixed-effects model (Mantel-Haenszel method). ${ }^{14,15}$ A random-effects model allows both between-study and within-study heterogeneities, whereas a fixed-effects model considers only within-study heterogeneity. To evaluate the association, four different ORs were calculated. At least two studies were required for estimating pooled effect sizes. Meta-analysis was carried out using the "metan" STATA command. Cochran's $Q$ test was used to assess statistical significance of between-study heterogeneity. Separated analysis was undertaken according to ethnicity. For sensitivity analysis, the potential influence of each individual study on the pooled estimation was examined by excluding each study in turn. Evidence for publication bias was assessed using the Begg's test. The "metabias" STATA command was used for publication bias evaluation.

\section{Results \\ Description of studies}

The study selection and exclusion process is outlined in Figure 1. Our initial literature search yielded a total of 


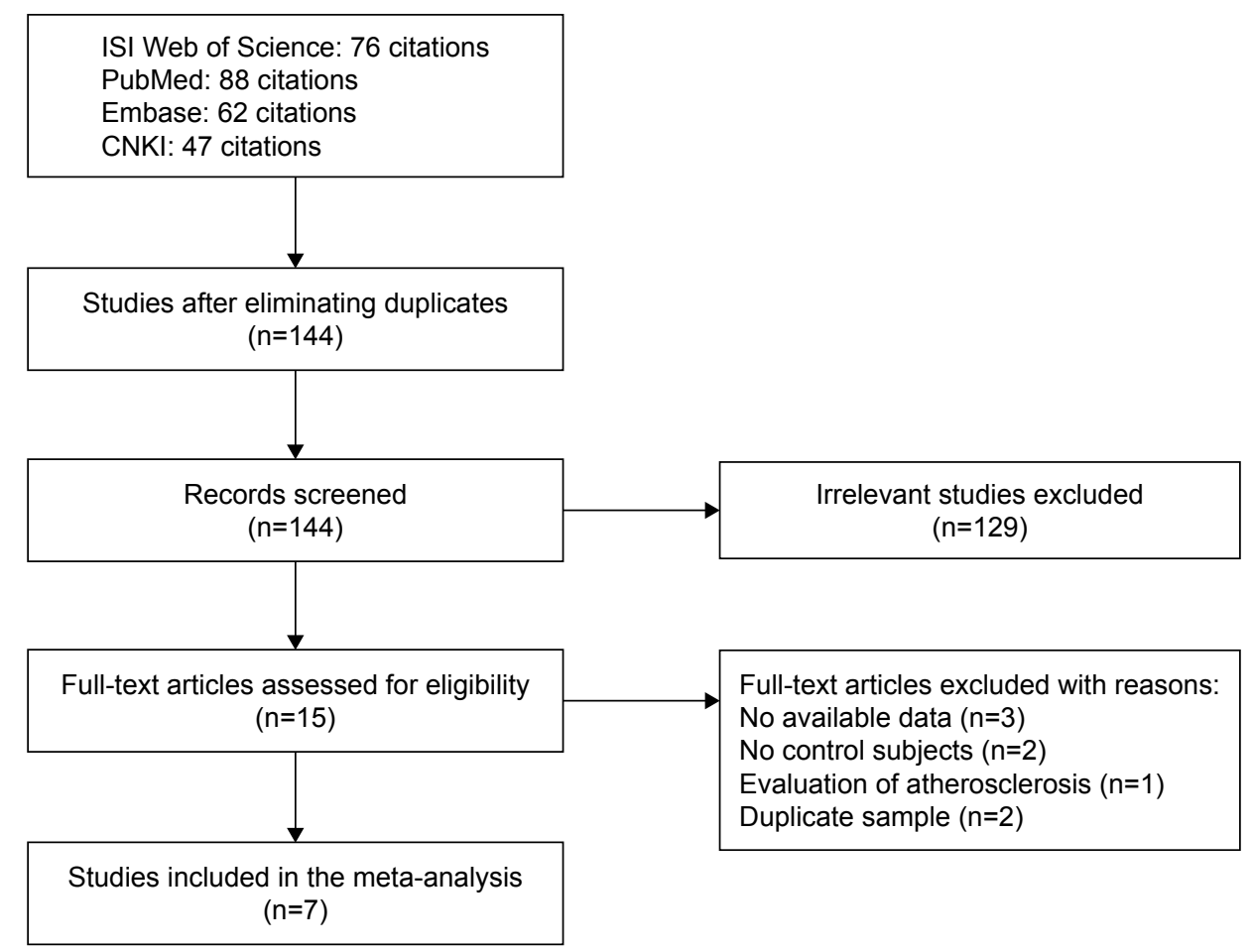

Figure I Flowchart depicting literature search and study selection.

Abbreviation: $\mathrm{CNKI}$, China National Knowledge Infrastructure.

273 studies using different combinations of keywords, of which 76 from ISI Web of Science, 62 from Embase, 88 from PubMed, and 47 from CNKI. After eliminating duplicates, 144 citations including titles and abstracts were screened for eligibility. Fifteen studies underwent a full text review, and eight studies were excluded because they did not meet the inclusion criteria. Finally, seven studies were included in the quantitative analysis. ${ }^{16-22}$ Among the eligible studies, six studies consisting of 1,840 cases and 1,816 controls assessed rs3025058, ${ }^{16,17,19-22}$ while two studies including 382 cases and 442 controls evaluated rs679620. ${ }^{18,19}$ All stroke patients underwent computerized tomography scan or magnetic resonance imaging of the brain. The characteristics of the eligible studies are presented in detail in Table 1. Genotype and allele frequencies of each polymorphism are summarized in Table 2.

\section{Meta-analysis of the MMP-3 polymorphisms and ischemic stroke risk} The rs679620 polymorphism was evaluated in Asian casecontrol association studies. ${ }^{18,19}$ There was no statistically significant evidence for heterogeneity between the studies (Table 3 and Figure 2). The results showed that the $M M P-3$ rs679620 A allele carriers had increased risk of ischemic stroke compared with homozygotes for the G allele (AA + GA vs GG: OR $=1.42,95 \%$ CI: $1.05-1.91, P=0.022$ ) (Table 3 and Figure 2). Similar results were found for the allele contrast (A allele vs $\mathrm{G}$ allele: $\mathrm{OR}=1.24,95 \% \mathrm{CI}: 1.00-1.54$, $P=0.047$ ) (Table 3 ) but not for the recessive (AA vs GA + GG: OR=1.14, 95\% CI: 0.76-1.73, $P=0.524)$ and homozygote (AA vs GG: OR=1.38, 95\% CI: 0.88-2.16, $P=0.161$ ) models (Table 3 ).

The rs3025058 polymorphism was assessed in Asian and Caucasian studies from 2002 to 2017. ${ }^{16,17,19-22}$ For all studies combined, no significant associations were found between rs3025058 genotypes and ischemic stroke risk (5A5A + 6A5A vs 6A6A: OR $=1.04,95 \%$ CI: $0.73-1.47 ; 5 \mathrm{~A} 5 \mathrm{~A}$ vs $6 \mathrm{~A} 5 \mathrm{~A}+6 \mathrm{~A} 6 \mathrm{~A}: \mathrm{OR}=1.14,95 \% \mathrm{CI}: 0.74-1.77 ; 5 \mathrm{~A} 5 \mathrm{~A}$ vs 6A6A: OR =1.11, 95\% CI: 0.68-1.80) (Table 3 and Figure 3). Similar results were found for the allele contrast (OR =1.06, 95\% CI: 0.79-1.41) (Table 3). We further performed subgroup analyses based on ethnicity to evaluate the association. We did not find a significant association between rs3025058 and ischemic stroke risk in Asians and Caucasians, respectively (Table 3 and Figure 3). Between-study heterogeneity was identified $(P<0.10)$, as shown in Table 3 . 


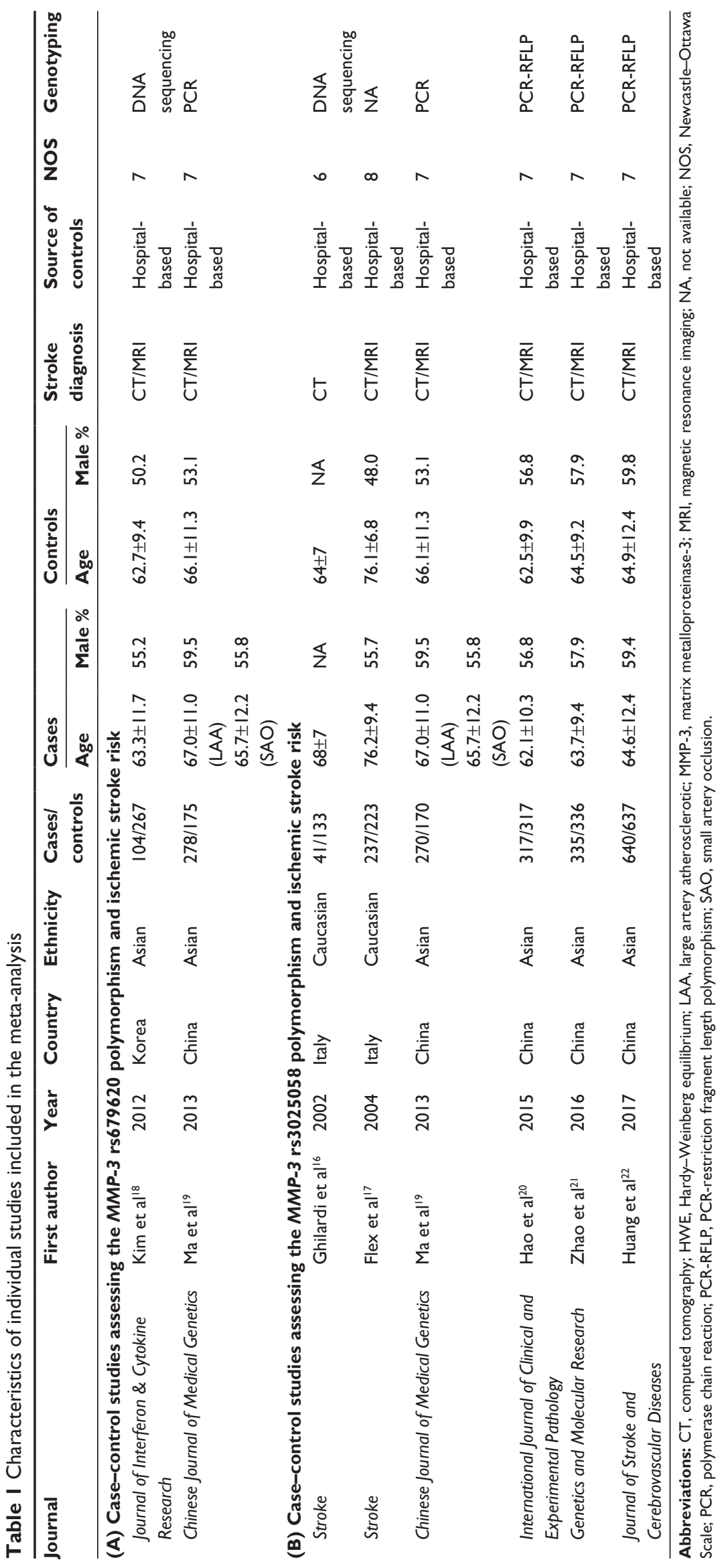






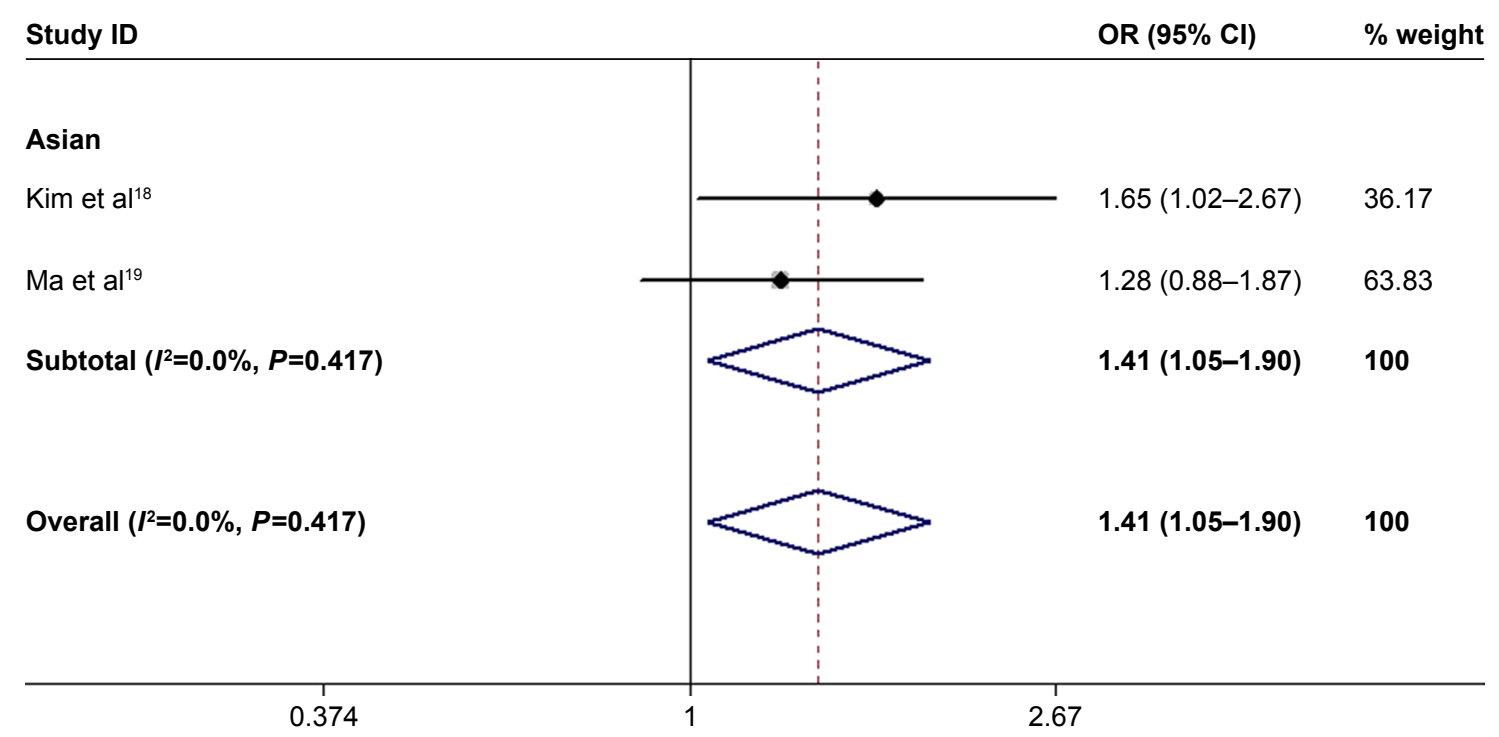

Figure 2 Forest plot for included studies examining the association between rs679620 and ischemic stroke risk under the dominant model (AA + GA vs GG). Abbreviation: OR, odds ratio.

\section{Sensitivity analysis and publication bias}

We did not perform sensitivity analysis for the rs679620 polymorphism, since there were only two eligible studies. Sensitivity analysis for the rs3025058 polymorphism (six eligible studies) showed that the significance of pooled ORs was not influenced excessively by omitting any single study (Figure 4). The Begg's test did not reveal any evidence of publication bias $(P>0.05)$.

\section{Discussion}

There have been inconsistencies in the results of case-control studies on the association of the $M M P-3$ polymorphisms rs679620 and rs3025058 with ischemic stroke. In order to verify the genetic contributions of these variants to ischemic stroke, we conducted a comprehensive literature search and pooled the results of independent association studies for meta-analysis. Our results showed that the rs679620 A allele

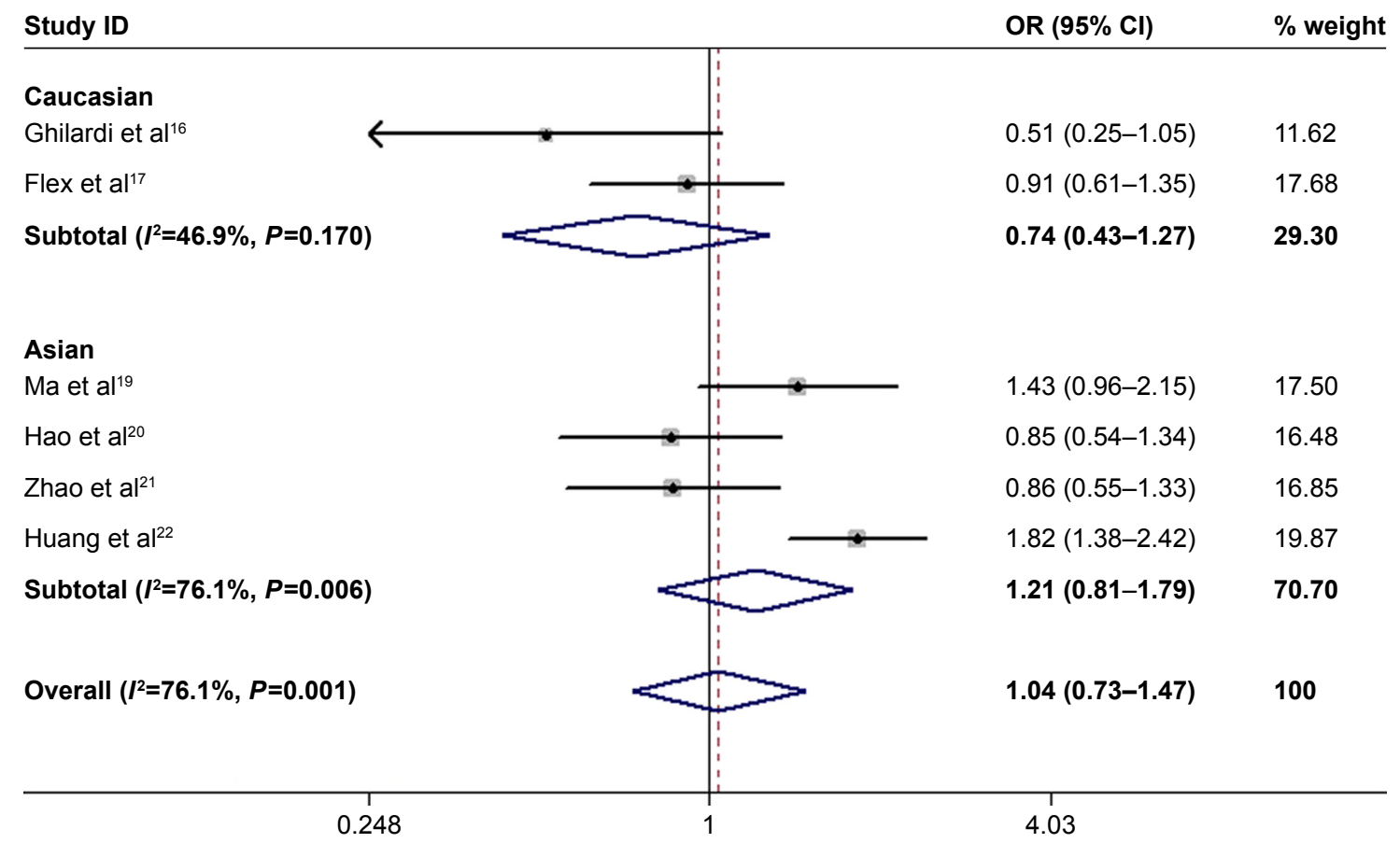

Figure 3 Forest plot for included studies examining the association between rs 3025058 and ischemic stroke risk (5A5A + 6A5A vs 6A6A). Note: Weights are from random-effects analysis.

Abbreviation: OR, odds ratio. 
Meta-analysis estimates, given named study is omitted

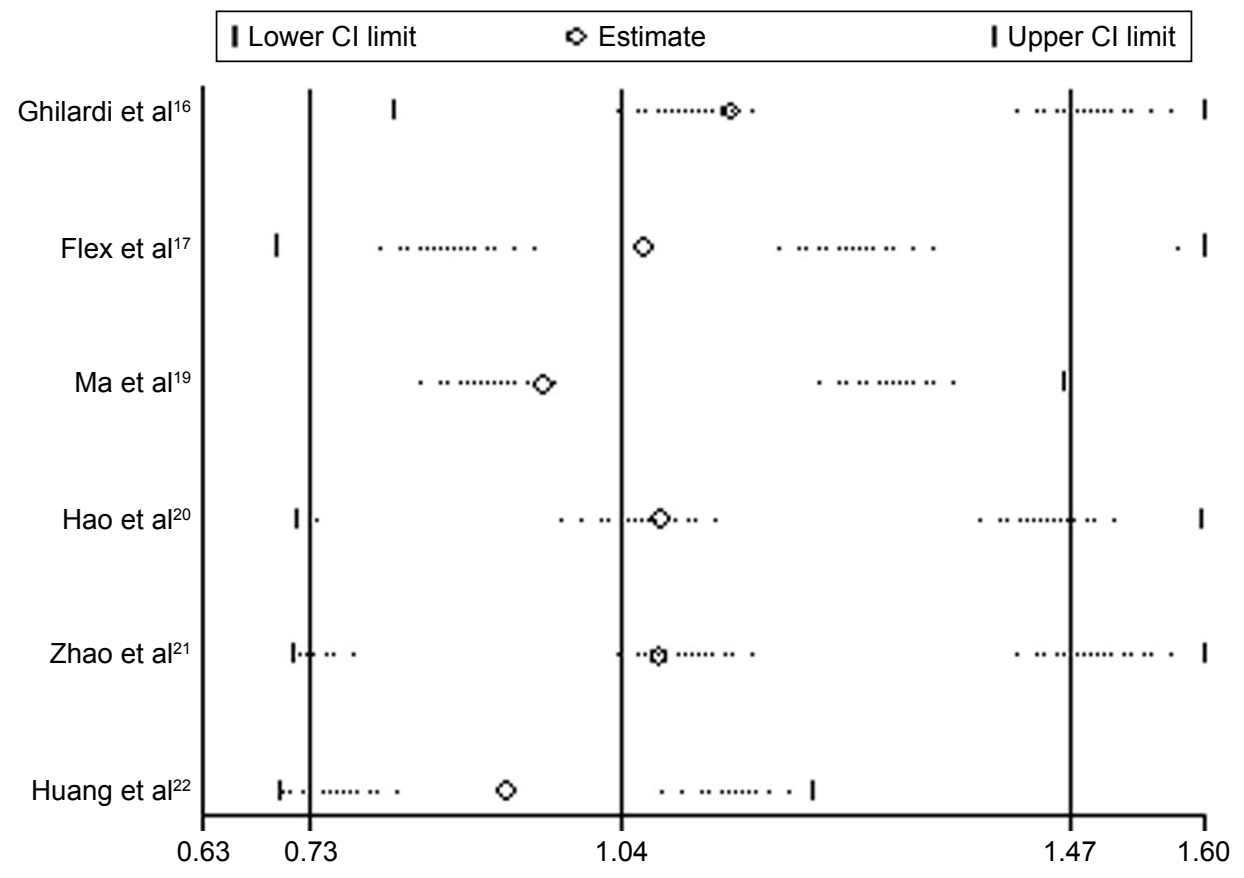

Figure 4 Sensitivity analysis for the association between rs 3025058 and ischemic stroke risk (5A5A + 6A5A vs 6A6A).

carriers had increased risk of ischemic stroke compared with homozygotes for the $\mathrm{G}$ allele in Asians, but there was no association between rs3025058 and ischemic stroke risk.

As far as we know, this is the first meta-analysis evaluating the association of rs679620 with ischemic stroke risk. Our results suggested that this polymorphism may be inherently involved in the pathogenesis of ischemic stroke and the A allele may be a possible risk allele. Concerning the rs3025058 polymorphism, our results were not consistent with those from a previous meta-analysis. ${ }^{23}$ In 2014, the study by Wen et $\mathrm{al}^{23}$ reported an association between rs 3025058 and ischemic stroke risk using a meta-analytic approach. However, there were several limitations in their results. First, patients with other diseases were included in the pooled estimation. The meta-analysis by Wen et al included eight studies for assessing the relation of rs3025058 with ischemic stroke risk. Besides studies on stroke patients, they included three irrelevant studies in the pooled analysis by mistake. The study by Djurić et $\mathrm{al}^{24}$ assessed the association between rs3025058 and carotid atherosclerosis in a Serbian population. The study by Buss et $\mathrm{al}^{25}$ evaluated the association between rs3025058 and cervical artery dissection, whereas the study by Fu et $\mathrm{al}^{26}$ investigated rs3025058 and the middle cerebral artery stenosis. None of the three studies provided data for assessing the association between rs3025058 and ischemic stroke risk. Obviously, it was not correct to include them as eligible studies. Second, data from the included studies were not carefully evaluated. The study by Ghilardi et al ${ }^{16}$ assessed the relationship of rs3025058 with carotid artery stenosis in 91 cases and 133 controls. Among the 91 patients with carotid artery stenosis, only 41 patients were suitable for the meta-analysis. However, Wen et al included all of the 91 carotid artery stenosis patients as stroke patients. Third, no subgroup analysis according to ethnicity was performed in the previous meta-analysis. Wen et $\mathrm{al}^{23}$ only evaluated the relationship of rs3025058 with ischemic stroke by combining all included studies. In contrast, in our meta-analysis, we not only assessed the overall association but also performed subgroup analysis in Caucasians and Asians, respectively. Because of the abovementioned limitations, the results of the previous meta-analysis were not accurate.

MMP-3 is a key member of the MMP family, with wide substrate specificity. ${ }^{7}$ It is capable of degrading many of the extracellular matrix components that are found within healthy and diseased blood vessels. It can also activate other enzymes in the MMP family, including MMP-1, MMP-8, MMP-9, and MMP-13. ${ }^{7}$ Human atheroma specimens have shown increased MMP-3 expression in atherosclerotic plaques' shoulders and regions of foam cell accumulation. ${ }^{9}$ A growing body of evidence suggests that increased expression of MMP-3 in atherosclerotic plaque results in increased plaque vulnerability and rupture, which is the main cause of 
ischemic stroke. ${ }^{27}$ In this meta-analysis, we found an association between rs679620 and ischemic stroke risk. rs679620 is located in exon 2 of the MMP-3 gene, leading to an amino acid change (Glu $>$ Lys) at residue 45 . It has been suggested that the amino acid change at this residue could have an effect on MMP-3 activation and function by modifying its interaction with other amino acids in this region. ${ }^{28}$ Given the important role of MMP-3 in atherosclerotic plaque inflammation and carotid atherosclerosis development, changes in MMP-3 activation and function induced by rs679620 may influence susceptibility to ischemic stroke.

This study has potential limitations. First, because of the limited availability of published results, the number of studies included in the meta-analyses for each polymorphism was relatively small. More well-designed case-control studies using larger sample sizes are needed in the future. Second, we did not have original data for all studies to evaluate gene-environment interaction. Although the meta-analysis did not find an association between rs3025058 and ischemic stroke risk, we did not exclude the possibility that an interaction between this polymorphism and other stroke risk factors including diabetes, smoking, and hypertension may affect ischemic stroke pathogenesis. Third, in our study, between-study heterogeneity was found in the comparisons for rs3025058. Differences in sample size, gender distribution, and genetic structure may contribute to the disparity in results. Other factors, such as environment and epigenetic change, may play roles in these differences as well. Fourth, the relation of the $M M P-3$ polymorphisms with ischemic stroke subtype was not assessed due to the paucity of data. Ischemic stroke has a number of subtypes with the most common being large-vessel atherosclerotic stroke, small-vessel disease, and cardioembolism. ${ }^{4}$ Evaluating the association of the $M M P-3$ polymorphisms with stroke subtypes may provide additional information. Unfortunately, the paucity of data precluded us the possibility to perform a pooled analysis for stroke subtypes.

\section{Conclusion}

The results of our meta-analysis suggest that the rs679620 A allele carriers have increased risk of ischemic stroke compared with homozygotes for the G allele in Asians, whereas there is no association between rs3025058 and ischemic stroke risk.

\section{Disclosure}

The author reports no conflicts of interest in this work.

\section{References}

1. Thrift AG, Thayabaranathan T, Howard G, et al. Global stroke statistics. Int J Stroke. 2017;12(1):13-32.

2. Morren JA, Salgado ED. Prevalence and control of stroke risk factors in a South Florida population. Int J Neurosci. 2012;122(12):734-741.

3. Mukherjee D, Patil CG. Epidemiology and the global burden of stroke. World Neurosurg. 2011;76(6 Suppl):S85-S90.

4. Hankey GJ. Stroke. Lancet. 2017;389(10069):641-654.

5. Traylor M, Farrall M, Holliday EG, et al. Genetic risk factors for ischaemic stroke and its subtypes (the METASTROKE collaboration): a metaanalysis of genome-wide association studies. Lancet Neurol. 2012;11: 951-962.

6. Rosenberg GA. Matrix metalloproteinases and their multiple roles in neurodegenerative diseases. Lancet Neurol. 2009;8:205-216.

7. Yamashita CM, Radisky DC, Aschner Y, Downey GP. The importance of matrix metalloproteinase-3 in respiratory disorders. Expert Rev Respir Med. 2014;8(4):411-421.

8. Henney AM, Wakeley PR, Davies MJ, et al. Localization of stromelysin gene expression in atherosclerotic plaques by in situ hybridization. Proc Natl Acad Sci U S A. 1991;88(18):8154-8158.

9. Galis ZS, Sukhova GK, Lark MW, Libby P. Increased expression of matrix metalloproteinases and matrix degrading activity in vulnerable regions of human atherosclerotic plaques. J Clin Invest. 1994; 94(6):2493-2503

10. Lien LM, Hsieh YC, Bai CH, et al. Association of blood active matrix metalloproteinase-3 with carotid plaque score from a community population in Taiwan. Atherosclerosis. 2010;212(2):595-600.

11. Taylor J, Sun YV, Chu J, et al. Interactions between metallopeptidase 3 polymorphism rs679620 BMI in predicting blood pressure in African-American women with hypertension. $J$ Hypertens. 2008;26: 2312-2318

12. Ye S, Eriksson P, Hamsten A, Kurkinen M, Humphries SE, Henney AM. Progression of coronary atherosclerosis is associated with a common genetic variant of the human stromelysin-1 promoter which results in reduced gene expression. J Biol Chem. 1996;271(22):13055-13060.

13. Stang A. Critical evaluation of the Newcastle-Ottawa Scale for the assessment of the quality of nonrandomized studies in meta-analyses. Eur J Epidemiol. 2010;25:603-605.

14. DerSimonian R, Laird N. Meta-analysis in clinical trials. Control Clin Trials. 1986;7(3):177-188.

15. Mantel N, Haenszel W. Statistical aspects of the analysis of data from retrospective studies of disease. J Natl Cancer Inst. 1959;22:719-748.

16. Ghilardi G, Biondi ML, DeMonti M, Turri O, Guagnellini E, Scorza R. Matrix metalloproteinase-1 and matrix metalloproteinase- 3 gene promoter polymorphisms are associated with carotid artery stenosis. Stroke. 2002;33(10):2408-2412.

17. Flex A, Gaetani E, Papaleo P, et al. Proinflammatory genetic profiles in subjects with history of ischemic stroke. Stroke. 2004;35(10): 2270-2275.

18. Kim SK, Kang SW, Kim DH, Yun DH, Chung JH, Ban JY. Matrix metalloproteinase-3 gene polymorphisms are associated with ischemic stroke. J Interferon Cytokine Res. 2012;32(2):81-86.

19. Ma AJ, Fan LY, Li WJ, et al. Association of matrix metalloproteinase-3 gene polymorphisms with subtypes of ischemic stroke. Chin JMed Genet. 2013;30:461-466.

20. Hao Y, Tian S, Sun M, Zhu Y, Nie Z, Yang S. Association between matrix metalloproteinase gene polymorphisms and development of ischemic stroke. Int J Clin Exp Pathol. 2015;8(9):11647-11652.

21. Zhao JH, Xu YM, Xing HX, et al. Associations between matrix metalloproteinase gene polymorphisms and the development of cerebral infarction. Genet Mol Res. 2016;14(4):19418-19424.

22. Huang XY, Han LY, Huang XD, Guan CH, Mao XL, Ye ZS. Association of matrix metalloproteinase-1 and matrix metalloproteinase-3 gene variants with ischemic stroke and its subtype. J Stroke Cerebrovasc Dis. 2017; 26(2):368-375. 
23. Wen D, Du X, Nie SP, Dong JZ, Ma CS. Association between matrix metalloproteinase family gene polymorphisms and ischemic stroke: a meta-analysis. Mol Neurobiol. 2014;50(3):979-985.

24. Djurić T, Zivković M, Radak D, et al. Association of MMP-3 5A/6A gene polymorphism with susceptibility to carotid atherosclerosis. Clin Biochem. 2008;41:1326-1329.

25. Buss A, Pech K, Roelver S, Bloemeke B, Klotzsch C, Breuer S. Functional polymorphisms in matrix metalloproteinases $-1,-3,-9$ and -12 in relation to cervical artery dissection. BMC Neurol. 2009;9:40.
26. Fu C, Xing Y, Song X. The association of the metalloproteinase- 3 gene promoter polymorphisms and the middle cerebral artery stenosis. Cell Biochem Biophys. 2011;59(3):185-189.

27. Clee SM. A role for MMP-3 genetic variation in atherosclerosis susceptibility? Atherosclerosis. 2010;208:30-31.

28. Chen Y, Nixon NB, Dawes PT, Mattey DL. Influence of variations across the MMP-1 and -3 genes on the serum levels of MMP-1 and -3 and disease activity in rheumatoid arthritis. Genes Immun. 2012;13(1) 29-37.

\section{Publish your work in this journal}

Neuropsychiatric Disease and Treatment is an international, peerreviewed journal of clinical therapeutics and pharmacology focusing on concise rapid reporting of clinical or pre-clinical studies on a range of neuropsychiatric and neurological disorders. This journal is indexed on PubMed Central, the 'PsycINFO' database and CAS, and is the official journal of The International Neuropsychiatric Association (INA). The manuscript management system is completely online and includes a very quick and fair peer-review system, which is all easy to use. Visit http://www.dovepress.com/testimonials.php to read real quotes from published authors.

Submit your manuscript here: http://www.dovepress.com/neuropsychiatric-disease-and-treatment-journal 